\title{
Using ocean colour remote sensing products to estimate turbidity at the Wadden Sea time series station Spiekeroog
}

S. P. Garaba

shungu.garaba@uni-oldenburg.de

\section{T. H. Badewien}

\section{A. Braun}

\section{A.-C. Schulz}

O. Zielinski

\begin{abstract}
Institute for Chemistry and Biology of the Marine Environment - Terramare, Carl von Ossietzky University of Oldenburg, Schleusenstraße 1, 26382 Wilhelmshaven, Germany

Institute for Chemistry and Biology of the Marine Environment - Terramare, Carl von Ossietzky University of Oldenburg, Schleusenstraße 1, 26382 Wilhelmshaven, Germany

Institute for Chemistry and Biology of the Marine Environment - Terramare, Carl von Ossietzky University of Oldenburg, Schleusenstraße 1, 26382 Wilhelmshaven, Germany

Institute for Chemistry and Biology of the Marine Environment - Terramare, Carl von Ossietzky University of Oldenburg, Schleusenstraße 1, 26382 Wilhelmshaven, Germany

Institute for Chemistry and Biology of the Marine Environment - Terramare, Carl von Ossietzky University of Oldenburg, Schleusenstraße 1, 26382 Wilhelmshaven, Germany
\end{abstract}

Time series measurements at the Wadden Sea time series station Spiekeroog (WSS) in the southern North Sea were used to empirically develop approaches for determining turbidity from ocean colour remote sensing products (OCPS). Turbidity was observed by a submerged optical sensor. Radiometric quantities were collected using hyperspectral radiometers. Surface reflected glint correction was applied to the radiometric quantities to compute remote sensing reflectance $\left(R_{R S}\right)$ and the $R_{R S}$ was converted into perceived colour of seawater matching the Forel-Ule colour Index (FUI) scale. The empirical approaches for determining turbidity from oCPs showed good least squares linear correlations and statistical significance $\left(R^{2}>0.7, p<0.001\right)$. These $0 C P$ approaches had relatively low uncertainties in predicting turbidity with encouraging mean absolute percent difference less than $31 \%$. The problem of bio-fouling on submerged sensors and the potential application of OCPS to monitor or correct for sensor drifts was evaluated. A protocol is proposed for the acquisition and processing of hyperspectral radiometric measurements at this optically complex station. Use of the classic FUI as a time series indicator of surface seawater changes did show promising results. The application of these OCPs in operational monitoring changes in water quality was also explored with the aim to evaluate the potential use of the wSS datasets in calibration and validation of satellite ocean colour remote sensing of these very turbid coastal waters.

[DOI: http://dx.doi.org/10.2971/jeos.2014.14020]

Keywords: Turbidity, ocean colour remote sensing, time series, water quality

\section{INTRODUCTION}

Wadden Sea time series station Spiekeroog (WSS, 53 $45.01644^{\prime} \mathrm{N}, 007^{\circ} 40.26552^{\prime} \mathrm{E}$ ) in Figure 1, located at the tidal inlet between islands Langeroog and Spiekeroog, is an operational hydrographic and marine environmental parameters observatory since autumn 2002 [1]. At the WSS measurements are available in near real-time, at 1-15 minute intervals, and with sensor maintenance carried out every fortnight depending on meteorological and sea conditions. Near real-time and continuous measurements are useful in operational monitoring and assist in rapid decision making with respects to water quality or marine ecosystems.

As more optical observations are becoming autonomous and continuous, it is critical to guarantee long term, traceable and high quality measurements $[2,3]$. We therefore need to take advantage of these (e.g. at WSS) frequent synoptic optical observations by developing approaches to infer and predict the surface water bio-optical state $[4,5]$. As there is a plethora of theoretical, semi-analytical, and empirical approaches linking optically active seawater constituents and physical oceanographic parameters (water quality indicators) to radiometric quantities or remote sensing reflectance $\left(R_{R S}\right)$ with good accuracy [6]-[8].

$R_{R S}$, a primary ocean colour product (OCP), is determined at the WSS station in a non-invasive manner using above-water hyperspectral radiometers (TriOS $\mathrm{GmbH}$, Germany). The surface reflected glint corrected $R_{R S}$ are transformed into the perceived Forel-Ule colour Index (FUI) scheme. The use of this FUI scheme is gaining interest especially in the European Union funded Citizens Observatory for Coast and Ocean Optical Monitoring (www.citclops.eu/) project. FUI is becoming more important because it is an OCP derived from the essential climate variable $R_{R S}$. It has recently become a standard output of a widely used radiative transfer numerical model Hydrolight (Sequoia Scientific Inc., USA) and has a long traceable history $[2,9,10]$. Other parameters (e.g. fluorescence, turbidity, oxygen, salinity) are observed in-water using autonomous optical sensors and are among typical marine environmental parameters used in water quality assessment as well as surveillance of such complex tidal inlet ecosystems. Turbidity is a measure of light scattering or attenuation capability of suspended particulate material 


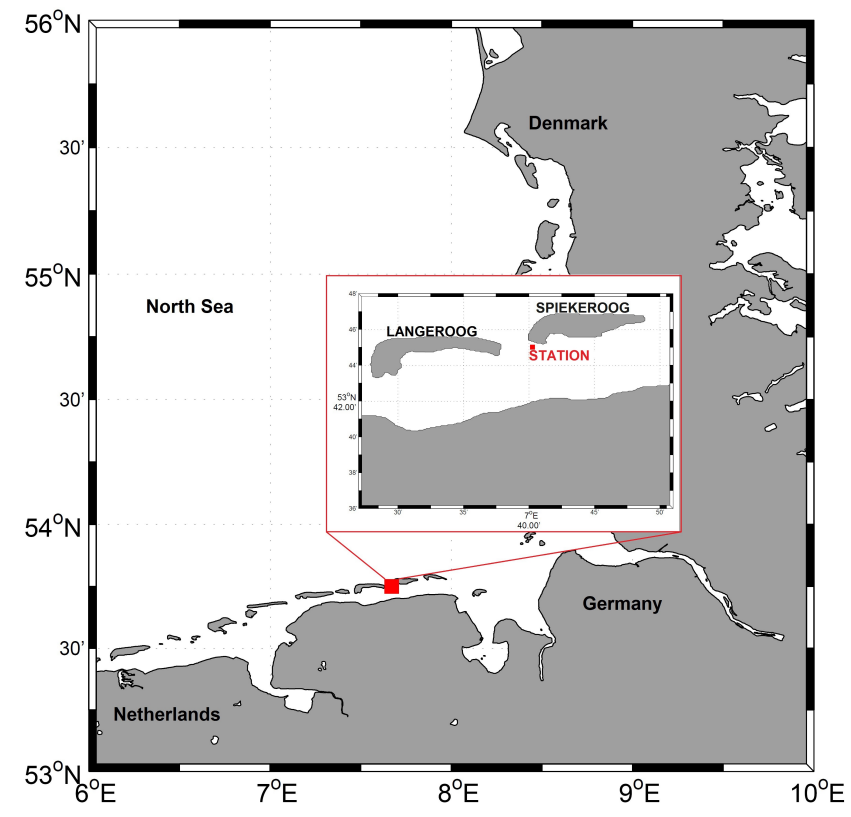

FIG. 1 Map showing the location of the Wadden Sea time series station Spiekeroog.

which affects seawater transparency and primary production [11]-[13]. It is therefore a very significant indicator of water quality that is related to OCPs and frequently measured in the ocean [14]-[17]. The goals of the study were; (i) to develop empirical bio-optical models or approaches for determining turbidity using OCPs obtained at the WSS station, (ii) present possible applications of OCPs to qualitatively monitor changes in marine environmental parameters, and (iii) as this is a long-term platform, present as well as propose a standard protocol for acquiring radiometric measurements, processing and useful end-products. To the best of our knowledge, no study has investigated remote sensing of turbidity in the Wadden Sea using hyperspectral radiometric quantities over such a frequent period. It therefore makes this study new and key to future investigations of turbidity from ocean colour. Remote sensing still faces the challenge of bio-optical algorithms that are region specific and not applicable to other regions $[6,8,16]$. We therefore aim to contribute to the time series hyperspectral ocean colour remote sensing in the Wadden Sea with additional marine environmental parameters. These parameters were collected between 14 August and 23 October 2013.

\section{METHODS}

\subsection{Above-water observations}

A radiometer setup, $24 \mathrm{~m}$ from the basin, with a TriOS RAMSES-ACC hyperspectral cosine irradiance meter for $E_{d}(\lambda)$ downwelling solar irradiance and two TriOS RAMSESARC hyperspectral radiance meters $L_{s f c}\left(\theta_{s f c}, \Phi_{S u n}, \lambda\right)$ for upwelling solar radiance and $L_{s k y}\left(\theta_{s k y}, \Phi_{S u n}, \lambda\right)$ for sky leaving radiance is in use at the WSS station. Figure 2 shows the top and side view of the sensor setup. The sky facing radiance sensor is set at $\theta_{s k y}=30^{\circ}$ zenith angle and the sea surface facing radiance sensor is set at $\theta_{s f_{c}}=30^{\circ}$ nadir angle and the azimuthal angle relative to North is $\Phi_{\text {North }}=159.5^{\circ}$.
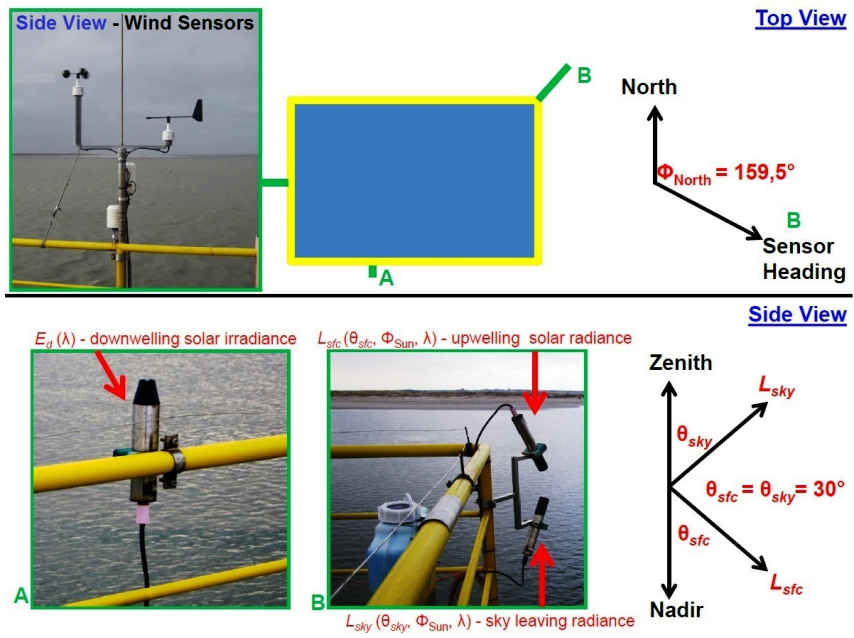

FIG. 2 Top and side view illustrations of TriOS RAMSES radiometers as well as Lambrecht wind sensors setup on the Wadden Sea time series station Spiekeroog.

$\Phi_{\text {Sun }}$ is the relative azimuthal angle of the radiance meters to the sun. The radiometers collect irradiance and radiance information continuously at 5 minute intervals. Wind speed is measured by a Lambrecht 14576 sensor cup anemometer and wind direction by Lambrecht 14566 sensor. These sensors are located $24 \mathrm{~m}$ above the basin. The calibrated radiometric measurements are stored and retrieved from TriOS MSDA_XE database for further processing in MathWorks MATLAB 2013a (code available on request via email). These calibrated measurements are available on request. Data processing involves a $5 \mathrm{~nm}$ binning over a spectral range $\lambda=(320-950) \mathrm{nm}$. Surface reflected glint correction was implemented as recommended in a standard protocol [14] to compute $R_{R S}$

$$
R_{R s}=\frac{L_{s f c}-L_{S R}}{E_{d}}
$$

where $E_{d}(\lambda)$ is downwelling solar irradiance, $L_{s f_{c}}\left(\theta_{s f_{c}}, \Phi, \lambda\right)$ is total upwelling sea surface radiance, $L_{S R}\left(\theta_{\text {sky }}, \Phi, \lambda\right)$ is the surface reflected glint. The surface reflected glint is part of the sky leaving radiance $L_{s k y}\left(\theta_{s k y}, \Phi, \lambda\right)$ and residual glint. Wind speed is used in the surface reflected glint correction to determine sea surface conditions.

\subsection{Forel-Ule Colours (FUI)}

The surface reflect glint corrected $R_{R S}$ spectra over the spectral range (380 - 720) $\mathrm{nm}$ are then converted into a single discrete FUI colour scale number using a colorimetric algorithm (Wernand, 2011). The scale has a colour index that matches perceivable seawater colours ranging from 1 (indigo-blue) to 21 (cola brown). The algorithm used transforms a spectrum by convolution of 1931 CIE-20-Colour Matching Functions (CMF) and $R_{R S}$ to obtain tristimuli values. Tristimuli values relate to the three primary colours: red, green, and blue. These tristimuli values are mapped on a chromaticity diagram matching discrete numerical numbers on the FUI scale. A full description of algorithm and background on the FUI is presented in literature $[9,18]$, the MATLAB code is available on request via email. 


\subsection{In-water observations}

An Eco FLNTU sensor (WETlabs, USA) determines chlorophyll fluorescence and turbidity at 1 minute intervals. The sensor is mounted $12 \mathrm{~m}$ above the basin, on the side of the pile diagonally opposite the radiometers, so that it is covered in water at low tide except at extreme low tides. Manufacturer provides calibration of the sensors and sensor cleaning is done fortnightly subject to weather conditions.

\subsection{Uncertainty analysis}

Correlations were explored between OCPs and turbidity. These observations are from different instruments we match and merge them according to time. Furthermore, to evaluate the performance of bio-optical model predictions we use in-situ turbidity observations as reference. The mean absolute percent difference $(M A P D)$

$$
M A P D=100 \% \times \frac{1}{N} \sum_{i=1}^{N} \frac{\left|X_{\text {predicted }}-X_{\text {in-situ }}\right|}{X_{\text {in-situ }}}
$$

where $N$ is the number of matching measurements, $X$ refers to turbidity that is observed in-situ and predicted by a model. In the case that the accurate value is unknown e.g. derived FUI, the mean unbiased percent difference $(M U P D)$ is computed

$$
M U P D=200 \% \times \frac{1}{N} \sum_{i=1}^{N} \frac{\left|X_{\text {measurement }_{1}}-X_{\text {measurement }_{2}}\right|}{X_{\text {measurement }_{1}}+X_{\text {measurement }_{2}}}
$$

\section{RESULTS AND DISCUSSION}

\subsection{Surface reflected glint correction and Forel-Ule index (FUI)}

In ocean colour, it is still a challenge to determine which surface reflected glint correction approach is superior $[2,14,19]$ We therefore use computed $R_{R S}$ from several widely used surface reflected glint correction approaches to objectively select a suitable correction approach. A total of 19889 spectra and their matching FUI plots measured between 14 August and 23 October 2013, example shown in Figure 3 and Figure 4 for 18 August 2013 at 11:00, were visually assessed in parallel. We perform visual inspection because in ocean colour there is no standard automated approach to distinguishing between good or bad spectra. We do not apply the meteorological flags for instance as in a previous study [20]. The available flags, e.g. masking spectra before sunrise and after sunset, are problematic as they are region specific and need to be adapted. Unfortunately adapting such flags requires auxiliary information (such as precipitation, fog, or minimal detectable solar radiation which is an arbitrary value) which is not available for this study. 9359 spectra remained after the visual inspection; this procedure eliminated contaminated, bad noisy spectra and those before sunrise and after sunset. For each computed $R_{R S}$ we derive the FUI colours from each surface reflected glint correction approach as presented in Figure 4. It is assumed that good spectra have minimal noise and a smooth signal, whilst spectra just before sunrise or just after sunset will have relatively more noise especially in the near-infrared and ultra-violet. The dark or at night spectra will have a flat

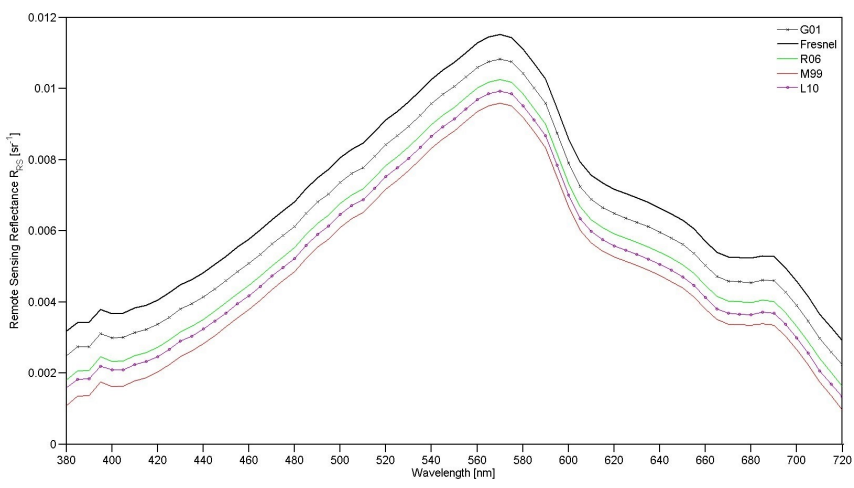

FIG. 3 Example of $R_{R S}$ after surface reflected glint correction observed on 18 August 2013 at 11:00. These correction approaches C01, R06, M99, and L10 are summarized in a recent protocol [14].
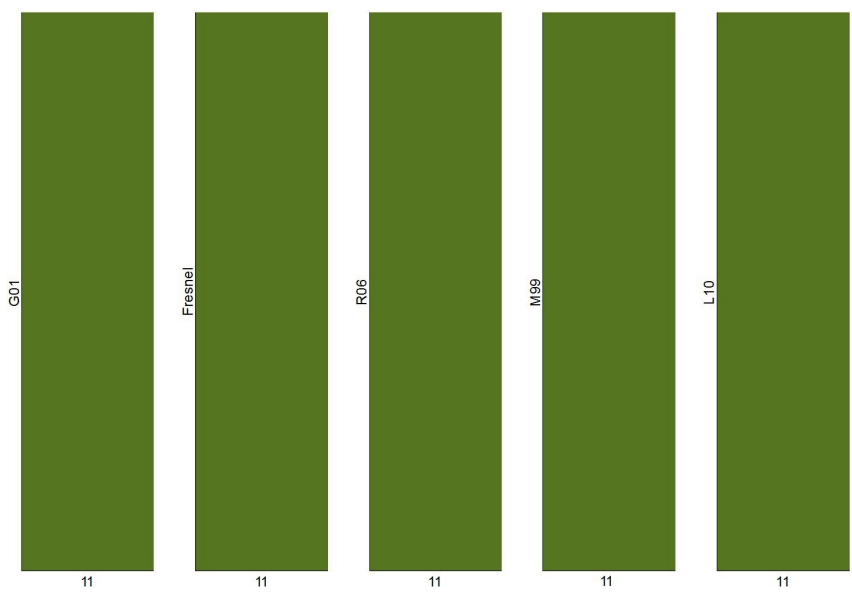

FIC. 4 Example of $R_{R S}$ derived Forel-Ule colours after surface reflected glint correction observed on 18 August 2013 at 11:00. These correction approaches G01, R06, M99, and L10 are summarized in a recent protocol [14].

signal with some spikes over the whole spectrum. Therefore, their derived FUI colours will show large variability as the algorithms depend on the spectrum shape. For instance, it is highly unlikely that at the time series station we will observe blue waters $(\mathrm{FUI}<3$ ) due to freshwater inflow laden with particulate material. To show this example spectra and derived FUI are presented in the Appendix showing observations after sunset/before sunrise. The goal of calculating FUI for all measurements is to provide a supplementary quality control tool. Furthermore, we aim to contribute to the large dataset of FUI as perceived colour of seawater is gaining interest in different natural science communities $[9,10]$. An example of how to quality control the $R_{R S}$ is to assume a valid measurement will have a similar derived FUI regardless of the surface reflected glint correction. With this quality control method, the spectral shape will be of interest. However, this method will be useless for other purposes e.g. bio-optical modelling that relies on band ratios or magnitude of $R_{R S}$. In our case, the assumed valid 9359 spectra had FUI values with MUPD less than $10 \%$. We assume that MUPD less than $10 \%$ accounts for the small differences in the surface reflected glint correction models.

As a colour of seawater monitoring tool, the average hourly changes over a week are shown in Figure 5. Ocean colour is limited by daylight and therefore we only present derived FUI 


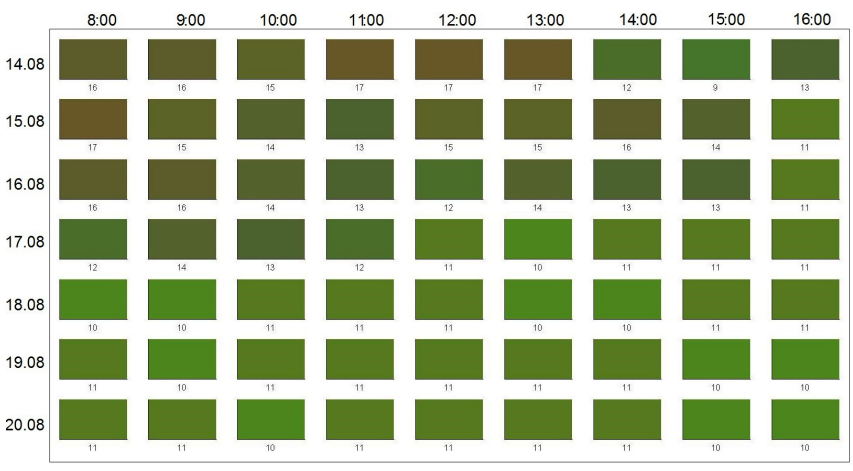

FIG. 5 An example application of ocean colour product (Forel-Ule colour Index) to monitor hourly averaged colour of seawater changes in between 08:00 to 16:00 from 14 and 20 August 2013.

colours between 8:00 and 16:00. It is therefore a useful tool for environmental water quality monitoring. Changes over a day or over a week can be observed and monitored as shown here.

\subsection{Turbidity and OCPS}

A total of 19889 turbidity observations from the Eco FLNTU sensor (WETlabs, USA) are evaluated. Figure 6 shows the raw turbidity observations. The main problem at the station is accumulation of biomaterial around the submerged sensors from bio fouling visible in Figure 6(b). Figure 6(a) observations of turbidity increase to 100 NTU because of bio fouling and soon after cleaning the measured turbidity decrease to values below 30 NTU. To determine the regional maximum turbidity observed we check with recent studies [17, 21, 22] and the maximum turbidity measured was 30 relative turbidity units. Furthermore, these turbidity observations of 30 relative turbidity units were also verified using the European Marine Ecosystem Observatory database (http://www. emecodata.net).

Therefore to estimate turbidity from radiometric observations we assume valid turbidity readings (i) are greater than 0 , (ii) less than the maximum 30 turbidity units, and (iii) within the 90 percentile of the 30 NTU i.e. less than 21 NTU. 2110 out of 19889 raw in-situ turbidity observations met this criterion.

We also noted that after each cleaning procedure the stable turbidity readings are $<30$ NTU as seen by observations after 09 October until 23 October 2013. It is important to take into consideration that this turbidity criterion here is empirical and would need to be adapted for each region accordingly. Were available the corresponding $R_{R S}$ spectra along with their derived FUI were paired and used to correlate the in-situ turbidity observations with the mean $R_{R S}$ in the spectral range (630 - 660) $\mathrm{nm}=R_{R S}(645 \mathrm{~nm})$ and their respective FUI. $R_{R S}(645$ $\mathrm{nm}$ ) is a common linearly correlated reference waveband for remote sensing of turbidity [15]-[17]. Furthermore, as surface reflected glint is a problem, to be objective in this study we test several surface reflected glint correction approaches and correlate them with turbidity. The best $R_{R S}(645 \mathrm{~nm})$ was that corrected after surface reflected glint approach G01 in [14], it had the least negative values and highest correlation coeffi-
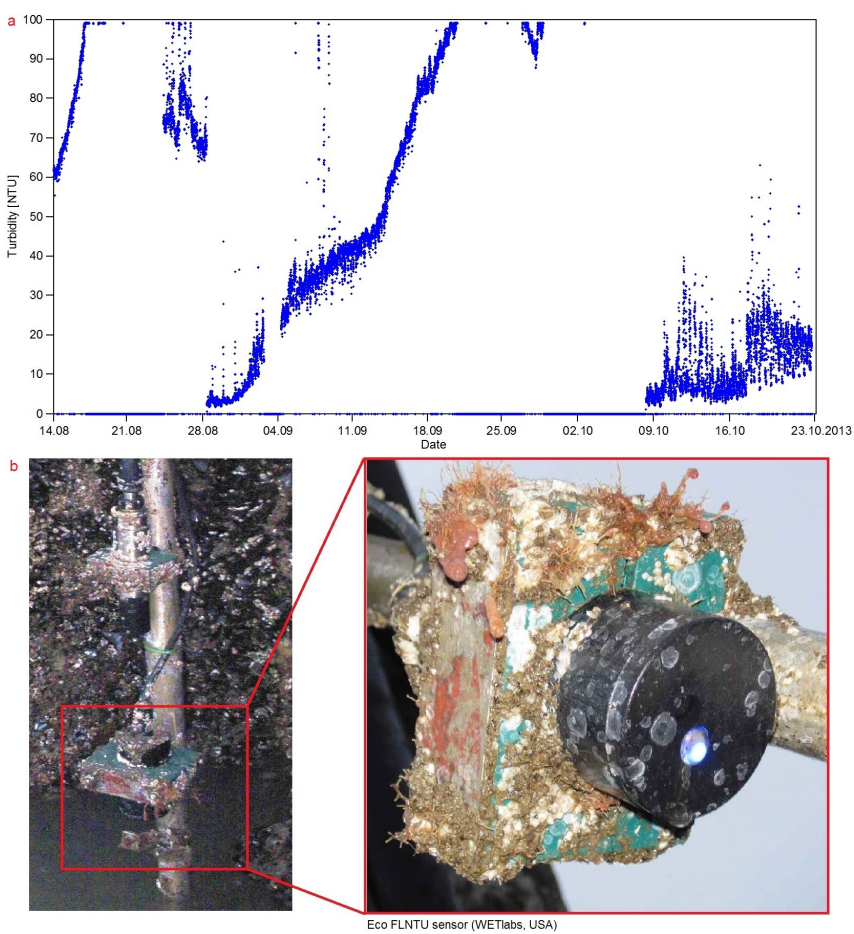

FIG. 6 a) Time series of all raw in-situ turbidity data between August and October 2013. b) Bio-fouling on the Eco FLNTU sensor (WETlabs, USA) sensors. As material starts to accumulate on the sensor window turbidity measurements increase rapidly for example after 04.09.2013, turbidity values reach 100 NTU which is sensor limit. After cleaning for example 09.10 .2013 to 23.10 .2013 measurements are below 30 NTU. Note the date format DD.MM.YYYY.

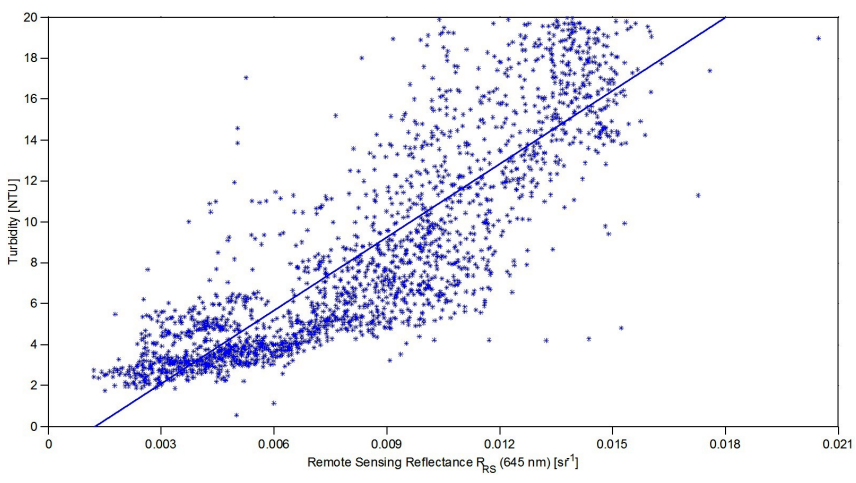

FIG. 7 Correlation between raw in-situ turbidity and mean $R_{R S}(645 \mathrm{~nm})$ between August and October 2013. The blue line represents a straight-line fit.

cient. The scatter plots of these findings are presented in Figure 7 and Figure 8.

The correlation between FUI and turbidity is presented in Figure 8 . The FUI scale consists of discrete numbers ranging from 1 to 21 and this could attribute to the clustering visible in Figure 8 . For instance, FUI $=8$ has both in-situ and corrected turbidity range $(2-3)$ NTU or FUI $=18$ has both in-situ and corrected turbidity range (13 - 19) NTU. To eliminate the clustering we extent the FUI scale by making it continuous the MATLAB code is available on request via email. There is a positive near linear relationship between turbidity and FUI. This is consistent with our expectation that as turbidity increases, water transparency decreases hence the perceived colour of seawater is expected to be darker i.e. high FUI values on the scale. Table 1 is a summary of the 


\begin{tabular}{|c|c|c|c|c|c|}
\hline Turbidity $=$ & $N$ & $R^{2}$ & $M S E$ & $M A P D$ & $p-$ value \\
\hline $1155.60 \times R_{R S}(645 \mathrm{~nm})-1.27$ & 2110 & 0.72 & 7.1 & $30.3 \pm 29.9$ & $<0.001$ \\
$1.52 \times \mathrm{FUI}_{\text {classic }}-11.03$ & 2110 & 0.77 & 5.9 & $25.9 \pm 27.2$ & $<0.001$ \\
$1.54 \times \mathrm{FUI}_{\text {modern }}-10.70$ & 2110 & 0.78 & 5.8 & $23.2 \pm 18.8$ & $<0.001$ \\
\hline
\end{tabular}

TABLE 1 Equations for estimating turbidity from $R_{R S}(645 \mathrm{~nm})$ or Forel-Ule Index (FUI) evaluated using $N$ stations to compute correlation coefficient $R^{2}$, mean square error $M S E$ error $\left[1 / N(y(i)-x(i))^{2}\right]$, mean absolute percent difference $M A P D$, and F-statistic $p-$ value

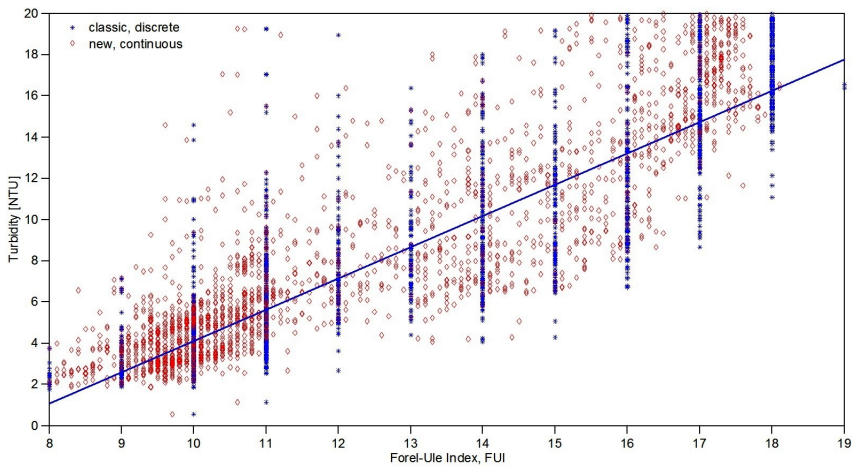

FIG. 8 Correlation between raw in-situ turbidity and Forel-Ule Index (FUI) between August and October 2013. The FUI is represented on the classic discrete scale and a modern continuous scale. The blue line represents a straight-line fit.

fit equations and statistics of plots in Figure 7 and Figure 8. The straight-line correlations show statistical significance with F-statistic $p$-value $<0.001$ and the correlation coefficient $\mathrm{R}^{2}>0.7$. We also evaluate a non-linear quadratic fit because in Figure 7 the data points exhibit a close to quadratic trend. The statistics are similar to those presented in Table 1, F-statistic $p$-value $<0.001, \mathrm{R}^{2}=0.75$. Here we show that there is a near linear relationship between hyperspectral radiometric measurements and in-situ turbidity. A better correlation can be determined by assuming a non-linear correlation. We also believe that the good correlations obtained from similar time series station in optically complex water bodies will help improve satellite remote sensing of coastal and turbid waters. The above-water OCPs can be used to validate and calibrate measurements from other platforms.

\section{CONCLUSION AND OUTLOOK}

The remote sensing of turbidity from OCPs at the Wadden Sea time series station Spiekeroog (WSS) is examined. A quality control approach is presented and involves the visual inspection of spectra and their matching FUI plots. The absence of a standard quality control protocol in ocean colour is accounted for by implementing several widely used surface glint correction approaches and objectively distinguishing them using steps and criterion proposed in recent investigations [2, 14]. Monitoring of water quality using the FUI colour scheme is also presented with relatively good potential application to natural sciences and policy makers.

We establish the relationship between hyperspectral OCPs and turbidity. The encouraging empirical correlations, in Table 1, suggests that OCPs can be used to validate the in-water turbidity meter or monitor its bio-fouling extent. We also show that there is potential in ocean colour remote

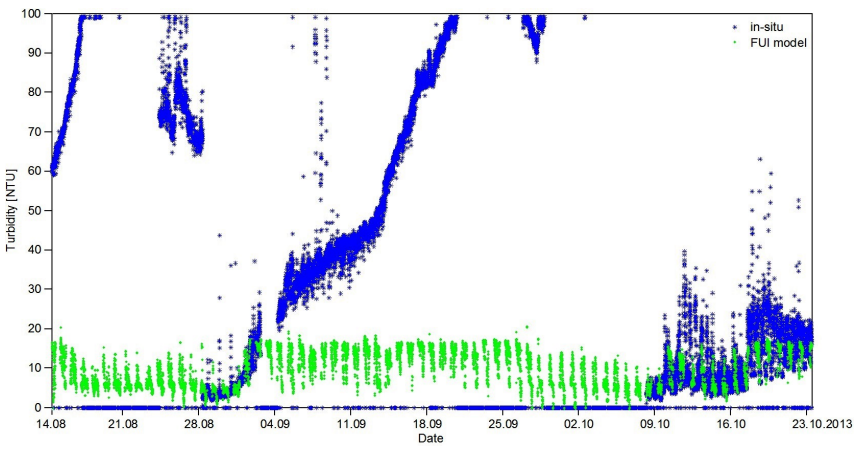

FIG. 9 Raw in-situ turbidity and Forel-Ule Index (FUI) estimated turbidity between August and October 2013. The FUI model used is given in Table 1 (FUI modern).

sensing of turbidity. FUI scale, an OCP of these hyperspectral radiometric measurements, is presented as a qualitative indicator of perceived colour of seawater. With caveats, it can also be used as a proxy for turbidity, considering its good correlation with turbidity presented in this study. The classic FUI scale definitely suffers from clustering since it has discrete values. However, the modern modified continuous does eliminate the clustering effect. It is therefore in our opinion a useful approximating tool for turbidity. As assessment and monitoring of marine ecosystems has become vital in climate change studies, we believe the FUI is important. We aim to take advantage of the vast near real-time WSS station data to contribute to the large dataset of FUI observations.

Figure 9 is an example of how we can use FUI to estimate turbidity in instances whereby the turbidity sensor is affected by bio-fouling. The turbidity is modelled from the FUI. The model predictions are subject to the number of FUI data available. In our opinion FUI is a useful parameter in correcting for bio fouling to some extent. It is important to take note that this is region specific and will vary for each region. To expand the robustness of turbidity monitoring over larger time scales our future research is to implement three independent approaches; (i) above-water ocean colour, (ii) in-situ turbidity meter, and (iii) bottom mounted upward looking Acoustic Doppler Current Profiler (ADCP) and relate them to turbidity as well as evaluate their covariances.

\section{ACKNOWLEDGMENTS}

We would like to thank Helmo Nicolai and his team for the continued support with station maintenance. Support from Coastal Observation System for Northern and Arctic Seas (COSYNA) is gratefully acknowledged. We are grateful to the comments from the anonymous reviewers. 


\section{A APPENDIX}

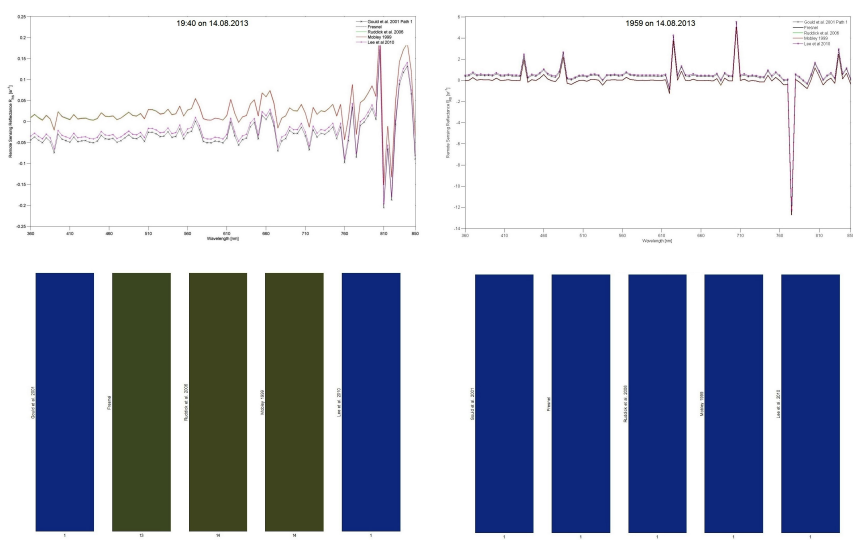

FIG. $10 \mathrm{~A}$ time series of spectra and derived Forel-Ule colours after surface reflected glint correction. In this example observations are at 19:40 and 19:59 on 14.08.2013. As light conditions are changing the spectrum becomes noisy and any derived product will be inconsistent or invalid.

\section{References}

[1] T. H. Badewien, E. Zimmer, A. Bartholomä, and R. Reuter, "Towards continuous long-term measurements of suspended particulate matter (SPM) turbid coastal waters," Ocean Dynam. 59, 227-238 (2009).

[2] S. P. Garaba, and 0. Zielinski, "Comparison of remote sensing reflectance from above-water and in-water measurements west of Greenland, Labrador Sea, Denmark Strait, and west of Iceland," Opt. Express 21, 15938-15950 (2013).

[3] C. Moore, A. Barnard, P. Fietzek, M. R. Lewis, H. M. Sosik, S. White, and 0 . Zielinski, "Optical tools for ocean monitoring and research," Ocean Sci. 5, 661-684 (2009).

[4] K. S. Baker, and R. C. Smith, "Bio-optical classification and model of natural waters. 2," Limnol. Oceanogr. 27, 500-509 (1982).

[5] R. Doerffer, F. Colijn, and J. Van Beusekom, Observing the Coastal Sea - an Atlas of Advanced Monitoring Techniques (LOICZ Reports \& Studies 33, 2008).

[6] F. Gohin, "Annual cycles of chlorophyll-a, non-algal suspended particulate matter, and turbidity observed from space and in-situ coastal waters," Ocean Sci. 7, 705-732 (2011).

[7] Z. P Lee, Remote Sensing of Inherent Optical Properties: Fundamentals, Tests of Algorithms, and Applications (Internationla Ocean-Colour Coordinating Group 5 (IOCCG 5), 2006).

[8] Y. Liu, M. A. Islam, and J. Gao, "Quantification of shallow water quality parameters by means of remote sensing," Prog. Phys. Geog. 27, 24-43 (2003).

[9] M. R. Wernand, Poseidons paintbox : historical archives of ocean colour in global-change perspective (PhD Thesis, Utrecht University, 2011).
[10] M. R. Wernand, H. J. van der Woerd, and W. W. C. Gieskes, "Trends in ocean colour and chlorophyll concentration from 1889 to 2000, worldwide," PLOS One 8, e63766 (2013).

[11] J. E. Cloern, "Turbidity as a control on phytoplankton biomass and productivity in estuaries," Cont. Shelf Res. 7, 1367-1381 (1987).

[12] R. J. Davies-Colley, and D. G. Smith, "Turbidity, suspended sediment, and water clarity: a review," J. Am. Water Resour. As. 37, 1085-1101 (2001).

[13] G. K. Moore, "Satellite remote sensing of water turbidity / Sonde de télémesure par satellite de la turbidité de l'eau," Hydrolog. Sci. Bulletin 25, 407-421 (1980).

[14] S. P. Garaba, and 0. Zielinski, "Methods in reducing surface reflected glint for shipborne above-water remote sensing," J. Europ. Opt. Soc. Rap. Public 8, 13058 (2013).

[15] F. N. Güttler, S. Niculescu, and F. Gohin, "Turbidity retrieval and monitoring of Danube Delta waters using multi-sensor optical remote sensing data: An integrated view from the delta plain lakes to the western-northwestern Black Sea coastal zone," Remote Sens. Environ. 132, 86-101 (2013).

[16] M. J. Moreno-Madrinan, M. Z. Al-Hamdan, D. L. Rickman, and F. E Muller-Karger, "Using the Surface Reflectance MODIS Terra Product to Estimate Turbidity in Tampa Bay, Florida," Remote Sens. 2, 2713-2728 (2010).

[17] B. Nechad, K. G. Ruddick, and G. Neukermans, "Calibration and validation of a generic multisensor algorithm for mapping of turbidity in coastal waters, in Bostater," in Proceedings of SPIE Volume: 7473 -Remote Sensing of the Ocean, Sea Ice, and Large Water Regions, 502-507 (SPIE, Berlin, 2009)

[18] H. M. Dierssen, R. M. Kudela, J. P. Ryan, and R. C. Zimmerman, "Red and black tides: Quantitative analysis of water-leaving radiance and perceived color for phytoplankton, colored dissolved organic matter, and suspended sediments," Limnol. Oceanogr. 51, 2646-2659 (2006).

[19] S. B. Hooker, G. Lazin, G. Zibordi, and S. McLean, "An evaluation of above- and in-water methods for determining water-leaving radiances," J. Atmos. Ocean. Tech. 19, 486-515 (2002).

[20] S. P. Garaba, J. Schulz, M. R. Wernand, and 0. Zielinski, "Sunglint detection for unmanned and automated platforms," Sensors 12, 12545-12561 (2012).

[21] G. Neukermans, K. G. Ruddick, and N. Greenwood, "Diurnal variability of turbidity and light attenuation in the southern North Sea from the SEVIRI geostationary sensor," Remote Sens. Environ. 124, 564-580 (2012).

[22] C. J. M. Philippart, M. S. Salama, J. C. Kromkamp, H. J. van der Woerd, A. F. Zuur, and G. C. Cadée, "Four decades of variability in turbidity in the western Wadden Sea as derived from corrected Secchi disk readings," J. Sea. Res. 82, 67-79 (2013). 